\title{
2. SYNOPSIS
}

\begin{tabular}{|c|c|c|}
\hline \multicolumn{2}{|c|}{ Name of Sponsor: I.R.I.S., 50 rue Carnot - 92284 Suresnes Cedex - France } & \multirow{2}{*}{$\begin{array}{l}\text { (For National } \\
\text { Authority Use only) }\end{array}$} \\
\hline $\begin{array}{l}\text { Test drug } \\
\text { Name of Finished Product: } \\
\text { Not applicable } \\
\text { Name of Active Ingredient: } \\
\text { S } 05520 \text { (Indapamide SR/Amlodipine) }\end{array}$ & & \\
\hline Individual Study Table Referring to Part of the Dossier & Volume: & Page: \\
\hline
\end{tabular}

Title of study: Safety and efficacy of fixed dose combination of Indapamide SR/Amlodipine in single-pill versus free dual therapy at the same dose over 12-week of treatment, with conditional titration based on the blood pressure control, in patients with mild to moderate uncontrolled essential hypertension. An international, multicentre, randomised, open-label study.

Protocol No.: CL3-05520-005

EudraCT No.: Not applicable

The description of the study protocol given hereafter includes the modifications of the substantial amendment to the protocol.

\section{Name of international coordinator: \\ Names of national coordinators:}

\section{Study centres:}

International, multicentre study $=13$ centres located in 2 countries included 154 patients: 6 centres in Russian Federation (90 patients included), 7 centres in Armenia (64 patients included).

\section{Publication (reference): Not Applicable}

\begin{tabular}{|c|c|}
\hline $\begin{array}{l}\text { Studied period: } \\
\text { Initiation date: } 26 \text { June } 2014 \text { (date of first visit first patient) }\end{array}$ & $\begin{array}{l}\text { Phase of development of the study: } \\
\text { III }\end{array}$ \\
\hline
\end{tabular}

Completion date: 26 January 2015 (date of last visit last patient)

\section{Objectives:}

The purpose of this study was to assess the effect in lowering office systolic blood pressure of fixed dose combination of Indapamide/Amlodipine (Ind/Aml) versus free combination at the same dose (Ind + Aml).

\section{Efficacy}

To assess the effect in lowering office systolic blood pressure of fixed-dose Indapamide SR $1.5 \mathrm{mg} /$ Amlodipine in single-pill versus free dual therapy at the same dose over 12-week of treatment, with conditional titration based on the blood pressure control, in patients with mild to moderate essential hypertension:

- To assess the effect in lowering office Systolic Blood Pressure (SBP), Diastolic Blood Pressure (DBP) and derivate parameters.

- After 6 weeks and after 12 weeks of treatment.

- After introduction of higher dose of the Ind/Aml fixed or free dose combination as compared to the previous doses.

- To assess the response to the treatment and normalisation of blood pressure at each visit.

- To compare the efficacy of both arms on Home Blood Pressure Monitoring (HBPM) parameters after 12 weeks.

- To assess the efficacy in lowering Home Blood Pressure and derived parameters of each dose of each arm after 6 weeks of exposure (after the up-titration) as compared to the previous dose.

- To assess the efficacy of both free and fixed dose combinations on the Home Blood Pressure Monitoring parameters at each visit until the study end (W12).

Safety

- To assess the safety of Ind/Aml fixed dose and free combination during the study and after 12 weeks of treatment.

\section{Adherence to treatment}

- To assess the patient treatment compliance both by Morisky Medication Scale (MMAS-8) and by investigational medicinal products accountability. 


\begin{abstract}
Methodology:
This study was an international, multicentre, randomised, open-label phase III, over a 12-week treatment period study, comparing single-pill fixed dose combination of Indapamide SR $1.5 \mathrm{mg} /$ Amlodipine versus free dual therapy by Indapamide SR $1.5 \mathrm{mg}$ and Amlodipine, in 150 patients with mild to moderate essential hypertension (Isolated Systolic hypertension and Systolo-diastolic hypertension), after 1 week of active run-in treatment (treatment used by the patient before the selection), or without any treatment (if not previously treated), with conditional titration based on blood pressure control. A fixed randomisation, stratified per centre was done. All patients included were proposed to participate in the HBPM part of the study.

This study was performed in strict accordance with Good Clinical Practice including the archiving of essential documents.
\end{abstract}

\title{
Number of patients:
}

Planned (office part): 150 patients (75 patients in each treatment group).

Included: 154 patients (77 patients in each treatment group).

HBPM part: 125 patients included (59 patients in the Ind/Aml group and 66 patients in the Ind + Aml group).

\section{Diagnosis and main criteria for inclusion:}

Men or women $\geq 18$ years old, with confirmed mild to moderate essential uncontrolled combined systolic and diastolic hypertension or isolated systolic hypertension (defined as SBP $\geq 140$ and $<180 \mathrm{mmHg}$ and DBP $<110 \mathrm{mmHg}$ at inclusion measured with a validated automatic device in supine position after at least 10 minutes of rest), treated more than one month before selection with any monotherapy at maximal dose, or any free or fixed-dose bi-therapy at initial dose and who in the investigators' opinion required a change in medication, either because of insufficient efficacy or poor tolerability: SBP $\geq 140$ and $<180 \mathrm{mmHg}$ and DBP $<110 \mathrm{mmHg}$ ), or patients currently non-treated for hypertension, more than 2 months without any treatment having $\mathrm{SBP} \geq 160$ and $<180 \mathrm{mmHg}$ and $\mathrm{DBP}<110 \mathrm{mmHg}$.

Results of laboratory examinations should be into the normal range and 12-lead electrocardiogram normal or without any clinically significant anomaly (Left ventricular hypertrophy authorised).

\section{Test drug:}

Indapamide SR $1.5 \mathrm{mg} /$ amlodipine $5 \mathrm{mg}$ (Ind/Aml) fixe dose single-pill combination (dose 1) administered orally with water as one tablet daily in morning (except on visit days where the patients had to take the treatment after blood pressure measurements and clinical examination). An uptitration was performed at W6 for non-controlled patients to the next dose of the treatment strategy: indapamide SR $1.5 \mathrm{mg} / \mathrm{amlodipine}$ $10 \mathrm{mg}$ (i.e. dose 2).

Batch Nos: L0049043 (Ind $1.5 \mathrm{mg} / \mathrm{Aml} 5 \mathrm{mg}$ ), L0049045 (Ind $1.5 \mathrm{mg} / \mathrm{Aml} 10 \mathrm{mg}$ ).

\section{Comparator:}

Indapamide SR $1.5 \mathrm{mg}$ and amlodipine $5 \mathrm{mg}$ (Ind + Aml) free dual therapy administered (dose 1) orally with water as one tablet daily each one, in morning, except on visit days where the patients had to take the treatment after blood pressure measurements and clinical examination. An uptitration was performed at W6 for non-controlled patients to the next dose of the treatment strategy: indapamide SR $1.5 \mathrm{mg}$ and amlodipine $10 \mathrm{mg}$ (i.e. dose 2).

\section{Duration of treatment:}

Run-in period (1 week): The run-in period was dedicated to confirm the essential uncontrolled hypertension under patient's previous treatment or for patient not treated.

Open-label randomised treatment (12 weeks): one visit at W6 and W12 were performed. At visit W6, all controlled patients (defined as SBP $<140 \mathrm{mmHg}$ and $\mathrm{DBP}<90 \mathrm{mmHg}$ ) remained on the same treatment and all non-controlled patients were up-titrated to the next dose of the treatment strategy. Patients who at the W6 visit had the SBP $\geq 180 \mathrm{mmHg}$ or $\mathrm{DBP} \geq 110 \mathrm{mmHg}$ (mean of the last 2 out of 3 measurements) were withdrawn from the study. 


\section{Criteria for evaluation: \\ Efficacy measurements:}

OFFICE part:

Primary efficacy criterion:

- Supine SBP.

Secondary criteria:

- Supine and standing DBP.

- Standing SBP at 3 min.

- Supine Mean Blood Pressure (MBP), defined as MBP = 2/3 DBP + 1/3 SBP.

- Supine Pulse Pressure (PP), defined as PP = SBP - DBP.

- Response to the treatment defined as SBP $<140 \mathrm{mmHg}$ and DBP $<90 \mathrm{mmHg}$ and/or SBP decrease $\geq 20 \mathrm{mmHg}$ from baseline and/or DBP decrease $\geq 10 \mathrm{mmHg}$ from baseline.

- Normalisation of blood pressure corresponding to the percentage of patients with $\mathrm{SBP}<140 \mathrm{mmHg}$ and DBP $<90 \mathrm{mmHg}$.

HBPM part:

- Mean home systolic blood pressure (HSBP) over the 4 days preceding the study visit and 4 days following the visit.

- Mean home diastolic blood pressure (HDBP) over the 4 days preceding the study visit and 4 days following the visit.

- Mean morning HSBP and HDBP over the 4 days preceding the study visit and 4 days after the visit.

- Mean evening HSBP and HDBP over the 4 days preceding the study visit and 4 days after the visit.

- Mean global/morning/evening home PP and Heart Rate (HR) over the 4 days preceding the visit and 4 days after the visit.

- Weekly variation of mean, global, morning and evening HSBP, HDBP and PP between visits.

- HBPM normalisation defined as $<135 / 85 \mathrm{mmHg}$ for statistical analysis.

\section{Safety measurements:}

- Emergent adverse events at each visit.

- Vital signs: weight (at selection and at W12 visit), height (at selection), supine and standing blood pressure to identify hypotension and orthostatic hypotension at each visit, and heart rate at each visit.

- Laboratory test:

- Complete laboratory examinations performed in fasting condition, at selection visit (or within 7 days after selection) and within 7 days before W12 visit: blood biochemistry (sodium, potassium, calcium, chloride, uric acid, urea, creatinine, creatinine clearance, fasting blood glucose levels, total protein, triglycerides, total cholesterol, High-Density Lipoprotein (HDL)-cholesterol, Low-Density Lipoprotein (LDL)-cholesterol, ASpartate (Amino) Transferase (ASAT), ALanine (Amino) Transferase (ALAT), Gamma-Glutamyl Transferase (GGT), alkaline phosphatase), haematology (haemoglobin, haematocrit, erythrocytes, neutrophils, basophils, eosinophils, lymphocytes, monocytes, leucocytes and platelets).

- Simplified laboratory test after 1 week (+/- 2 days) of treatment (sodium, potassium, uric acid, creatinine and creatinine clearance, ALAT and ASAT).

- 12-lead electrocardiogram at W0 and W12 visit.

\section{Other measurements:}

- Patient's adherence to the treatment using the Morisky Medication Scale (MMAS-8).

Statistical methods:

Study outcome and safety analysis: descriptive statistics were provided.

Efficacy analysis:

The analysis Set used for the main analysis of the primary endpoints was the Full Analysis Set (FAS): based on the intention-to-treat principle, this set corresponded to randomised patients who received at least one dose of study treatment and who have at least one baseline analysable value and one post baseline analysable value for Supine SBP. 


\section{Statistical methods (Cont'd): \\ Efficacy analysis (Cont'd):}

For supine SBP, control BP and response rate parameters, analyses were done on all patients of the population. For supine DBP, MBP and PP parameters, analyses were done separately on Systolo-Diastolic Hypertension (SDH) and Isolated Systolic Hypertension (ISH) subgroups.

Primary endpoint: office SBP. The primary analysis was to study the efficacy of a single-pill combination versus free dual therapy, after a 12-week treatment period. A between-group difference was assessed on the change of supine SBP from baseline to last post baseline value, using a single two-way analysis of covariance (ANCOVA) model over the W0-W12 period. The model included the fixed categorical effects of treatment and country and the continuous fixed covariate of baseline. The between-group estimate (standard error) and two-sided 95\% Confidence Interval of the estimate were provided.

Secondary analyses: the between-group difference of the change from baseline to W6/W12 value of SBP was assessed with the same model used in the main analysis, and the within-group difference was assessed using Paired Student's test.

\section{Secondary criteria:}

- All analyses performed on the primary endpoint were carried out on all secondary quantitative parameters (Supine DBP, standing SBP and DBP at 3 min, MBP and PP).

- The between-group difference of proportions of controlled BP patients (respectively responder patients) based on last post baseline value was assessed using chi square test in W0-W12 and W0-W6 periods.

- HBPM part: all analyses performed on the primary endpoint (office SBP) were carried out on these HBPM quantitative parameters, except for daily / weekly variations of mean HSBP, HDBP and HPP. The within-group difference on change from baseline to each post-baseline value (W6 and W12) and change from W6 visit to W12, for titration effect was studied only over the 4 days preceding the study visit. The between-group difference of proportions of controlled BP patients based on last post baseline value was assessed using chi square test in W0-W12 and W0-W6 periods.

\section{SUMMARY - CONCLUSIONS}

\section{DISPOSITION OF PATIENTS AND ANALYSIS SETS}

Disposition of patients

\begin{tabular}{|c|c|c|c|c|}
\hline Status & & Ind/Aml & Ind + Aml & All \\
\hline Included/randomised & $\mathbf{n}$ & $77(100)$ & 77 (100) & $154(100)$ \\
\hline In compliance with the protocol & $\mathrm{n}(\%)$ & $69(89.6)$ & $63(81.8)$ & $132(85.7)$ \\
\hline With a protocol deviation before or at inclusion & $\mathrm{n}(\%)$ & $8(10.4)$ & $14(18.2)$ & $22(14.3)$ \\
\hline Withdrawn due to & n (\%) & $2(2.6)$ & $2(2.6)$ & $4(2.6)$ \\
\hline Adverse event & $\mathrm{n}(\%)$ & $1(1.3)$ & $1(1.3)$ & $2(1.3)$ \\
\hline Non-medical reason & $\mathrm{n}(\%)$ & - & $1(1.3)$ & $1(0.6)$ \\
\hline Other protocol withdrawal criteria & $\mathrm{n}(\%)$ & 1 & - & $1(0.6)$ \\
\hline Completed & n (\%) & $75(97.4)$ & $75(97.4)$ & $150(97.4)$ \\
\hline In compliance with the protocol & $\mathrm{n}(\%)$ & $75(97.4)$ & $75(97.4)$ & $150(97.4)$ \\
\hline With a protocol deviation after inclusion & $\mathrm{n}(\%)$ & - & - & - \\
\hline Full Analysis Set (FAS) & n (\%) & $77(100)$ & 77 (100) & $154(100)$ \\
\hline Per Protocol Set (PPS) & n $(\%) *$ & $74(96.1)$ & $71(92.2)$ & $145(94.2)$ \\
\hline Safety Set (SS) & n $(\%)$ & $77(100)$ & $77(100)$ & $154(100)$ \\
\hline
\end{tabular}

$\%$ : expressed as percentage of the included/randomised patients

*: expressed as percentage of the FAS

A total of 154 patients were included, with a well-balanced distribution reached (77 patients in the Ind/Aml group and 77 patients in the Ind + Aml group). Of them, 4 patients were withdrawn (2 in each treatment group), of whom half were due to adverse events. Finally, 150 patients, $97.4 \%$ of the included patients completed the study (75 in each treatment group). Overall, 22 patients, $14.3 \%$ reported protocol deviations before or at inclusion, with a lower frequency of patients affected in the Ind/Aml than Ind + Aml group ( 8 patients, $10.4 \%$ versus 14 patients, $18.2 \%$ ). They were mainly in relation with the sampling form: 6 patients, $7.8 \%$ in the Ind/Aml group and 7 patients, 9.1\% in the Ind + Aml group. After inclusion, one patient, $1.3 \%$ in the Ind/Aml group had one protocol deviation (blood sample not taken while the patient was withdrawn).

In the HBPM part of the study, 125 patients were included (59 patients in the Ind/Aml group and 66 patients in the Ind + Aml group). 


\section{SUMMARY - CONCLUSIONS (Cont'd) \\ BASELINE CHARACTERISTICS}

Demographic and other baseline characteristics of the patients fulfilled with the selection/inclusion criteria of the study protocol. In the Randomised Set, patients were in average $58.4 \pm 10.6$ years old, and most of them $(70.1 \%)$ were between 18 and 64 years old. More than half of the patients were women (105 patients, 68.2\%). BMI was on average $28.2 \pm 2.7 \mathrm{~kg} / \mathrm{m}^{2}$, with $33.1 \%$ of the patients that had a BI $\geq 30 \mathrm{~kg} / \mathrm{m}^{2}$.

All patients presented at selection an essential hypertension in average for $110.6 \pm 91.5$ months.

Most of the patients (142 patients, 92.2\%) received at least one treatment for essential hypertension before entry in the study, mainly Agents acting on the renin-angiotensin system (67 patients, $87.0 \%$ versus 69 patients, $89.6 \%$, respectively), and Diuretics (21 patients, 27.3\% versus 28 patients, 36.4\%, respectively). More than half of the patients $(57.0 \%)$ were on monotherapy. No relevant between-group difference was observed. Most of the patients (141 patients, 91.6\%) reported a medical history other than essential arterial hypertension, mainly related to Metabolism and nutrition disorders (46 patients, $59.7 \%$ in the Ind/Aml group and 58 patients, $75.3 \%$ in the Ind + Aml group), including mainly dyslipidaemia (37 patients, $48.1 \%$ versus 34 patients, $44.2 \%$, respectively). Regarding risk factors, $17.5 \%$ had smoking habit with a larger frequency reported in the Ind/Aml group than Ind + Aml group (22.1\% versus $13.0 \%$, respectively), and $20.1 \%$ had alcohol habit consumption, with no relevant between-group difference.

Among the 137 patients that performed an ECG at baseline, one patient in the Ind + Aml group had an ECG abnormality considered as clinically significant (left ventricular hypertrophy).

In the FAS, at baseline, the supine SBP and DBP were in average for overall patients: $154.03 \pm 8.49 \mathrm{mmHg}$ and $90.34 \pm 9.80 \mathrm{mmHg}$, supine PP: $63.69 \pm 12.40 \mathrm{mmHg}$ and supine MBP: $111.57 \pm 7.34 \mathrm{mmHg}$, with no relevant between group-difference. Standing SBP and DBP at $3 \mathrm{~min}$ were similar in both treatment groups at inclusion (153.1 $\pm 10.9 \mathrm{mmHg}$ for standing SBP and $91.8 \pm 10.8 \mathrm{mmHg}$ for standing DBP).

Demographic and other baseline characteristics were globally similar in the other Sets and showed same trends in patients who participated in the HBPM part.

\section{EXTENT OF EXPOSURE}

The global compliance (mean \pm SD) over W0-W12 was $98.9 \pm 6.3 \%$, with similar frequency in both treatment groups, in the Randomised Set and in the FAS. All patients except one had an overall compliance between $70 \%$ and $130 \%$ (inclusive). In the same way, the MMAS-8 showed a good global compliance over W0-W12 with a mean of $6.60 \pm 1.01$ over a maximal score of 8 . No relevant between-group difference was observed for the global compliance and the MMAS-8.

In total, 96 patients, $62.3 \%$ of the randomised patients remained treated with the dose 1 during the overall study duration (45 patients, $58.4 \%$ in the Ind $1.5 \mathrm{mg} / \mathrm{Aml} 5 \mathrm{mg}$ group and 51 patients, $66.2 \%$ in the Ind $1.5 \mathrm{mg}$ + Aml $5 \mathrm{mg}$ group), and 55 patients, 35.7\% (30 patients, 39.0\% and 25 patients, 32.5\%, respectively) were up-titrated to the dose 2 (Ind $1.5 \mathrm{mg} / \mathrm{Aml} 10 \mathrm{mg}$, and Ind $1.5 \mathrm{mg}+\mathrm{Aml} 10 \mathrm{mg}$ ), in the Randomised Set and the FAS. In addition, 3 patients prematurely withdrawn received the dose 1 over W0-W6. 


\section{SUMMARY - CONCLUSIONS (Cont'd) \\ EFFICACY RESULTS \\ Office part of the study}

\section{Primary efficacy endpoint: office supine systolic blood pressure}

Primary analysis: change in the office supine SBP from baseline to the last post-baseline value at W12

Over the W0-W12 period, the supine SBP decreased from baseline to the last post-baseline value at W12 in both treatment groups, in the FAS. The changes were similar in both treatment groups $(\mathrm{E}(\mathrm{SE})=0.79(2.03) \mathrm{mmHg}, 95 \% \mathrm{CI}=[-3.22 ; 4.79])$. Similar results were obtained in the PPS.

Office supine SBP ( $\mathrm{mmHg}$ ) - Change from baseline to last post-baseline value Comparison between groups - Over W0-W12 period - FAS $(\mathbf{N}=154)$

\begin{tabular}{lccc}
\hline Office supine SBP (mmHg) & & $\begin{array}{c}\text { Ind/Aml } \\
(\mathbf{N}=\mathbf{7 7})\end{array}$ & $\begin{array}{c}\text { Ind + Aml } \\
(\mathbf{N}=\mathbf{7 7})\end{array}$ \\
\hline \multirow{3}{*}{ Baseline } & $\mathrm{n}$ & 77 & 77 \\
& Mean \pm SD & $153.52 \pm 7.84$ & $154.54 \pm 9.11$ \\
& $95 \%$ CI & {$[151.74 ; 155.30]$} & {$[152.47 ; 156.61]$} \\
END W12 & Min ; Max & $142.0 ; 171.5$ & $140.5 ; 177.0$ \\
& Mean \pm SD & $133.18 \pm 13.51$ & $132.73 \pm 12.31$ \\
& $95 \%$ CI & {$[130.11 ; 136.25]$} & {$[129.94 ; 135.53]$} \\
END W12 - Baseline & Min ; Max & $102.0 ; 171.5$ & $107.5 ; 160.5$ \\
& Mean \pm SD & $-20.34 \pm 13.42$ & $-21.81 \pm 13.84$ \\
& $95 \%$ CI & {$[-23.38 ;-17.29]$} & {$[-24.95 ;-18.67]$} \\
& Min; Max & $-45.0 ; 18.0$ & $-58.5 ; 6.5$
\end{tabular}

Statistical analysis

Parametric approach with adjustment

$$
\begin{array}{cc}
\text { E (SE) (1) } & 0.79(2.03) \\
95 \% \text { CI }(2) & {[-3.22 ; 4.79]}
\end{array}
$$

$N$ : total number of patients in each treatment group; $n$ : number of patients affected

(1) Estimate (Standard Error) of the difference in adjusted mean changes from baseline to last post-baseline value until W12 indapamidelamlodipine - indapamide + amlodipine using a General Linear Model with treatment, baseline and country as covariates

(2) $95 \%$ Confidence Interval of the estimate

\section{Secondary analyses}

Secondary analyses showed same trends in the FAS. An important decrease was observed in the office SBP in each treatment group from baseline to last post-baseline value over each treatment period:

- W0-W6 period:

- Ind/Aml group: $-17.65 \pm 12.99 \mathrm{mmHg}(\mathrm{E}(\mathrm{SE})=-17.65(1.48) \mathrm{mmHg}, 95 \% \mathrm{CI}=[-20.60 ;-14.70])$

- Ind + Aml group: $-20.46 \pm 14.17 \mathrm{mmHg}(\mathrm{E}(\mathrm{SE})=-20.46(1.62) \mathrm{mmHg}, 95 \% \mathrm{CI}=[-23.68 ;-17.24])$

- Between-group analysis: $\mathrm{E}(\mathrm{SE})=2.31(2.10) \mathrm{mmHg}, 95 \% \mathrm{CI}=[-1.85 ; 6.46]$

- W0-W12 period:

- Ind/Aml group: $-20.34 \pm 13.42(\mathrm{E}(\mathrm{SE})=-20.34(1.53) \mathrm{mmHg}, 95 \% \mathrm{CI}=[-23.38 ;-17.29])$

- Ind+Aml group: $-21.81 \pm 13.84(\mathrm{E}(\mathrm{SE})=-21.81(1.58) \mathrm{mmHg}, 95 \% \mathrm{CI}=[-24.95 ;-18.67])$

Over the W6-W12 period, additional decreases were observed in each treatment group $(-2.76 \pm 13.07 \mathrm{mmHg}$ in the Ind/Aml group versus $-1.36 \pm 12.24 \mathrm{mmHg}$ in the Ind + Aml group).

When considering only patients not controlled at W6 ( $\mathrm{n}=55$ patients), the evaluation of the up-titration efficiency showed that the titration allowed an important decrease over W6-W12:

- Ind/Aml group: $-10.85 \pm 9.20 \mathrm{mmHg}(\mathrm{E}(\mathrm{SE})=-10.85(1.68) \mathrm{mmHg}, 95 \% \mathrm{CI}=[-14.29 ;-7.41])$

- Ind + Aml group: $-9.86 \pm 10.33 \mathrm{mmHg}(\mathrm{E}$ (SE) $-9.86(2.07) \mathrm{mmHg}, 95 \% \mathrm{CI}=[-14.13 ;-5.59]$. 


\section{SUMMARY - CONCLUSIONS (Cont'd) \\ EFFICACY RESULTS (Cont'd) \\ Secondary efficacy endpoints}

Results of the secondary efficacy endpoints were in the same line than those described for the primary efficacy endpoint.

\section{- Office supine DBP}

Over the W0-W12 period, in both treatment groups an important decrease in the office supine DBP was observed in both treatment groups from baseline to the last post-baseline value. The decrease was similar in both treatment groups, and as expected, marked decreases were detected only in patients with a systolic-diastolic hypertension at inclusion:

- FAS SDH: $-15.18 \pm 9.58 \mathrm{mmHg}$ in the Ind/Aml group versus $-11.73 \pm 8.14 \mathrm{mmHg}$ in the Ind $+\mathrm{Aml}$ group $(\mathrm{E}(\mathrm{SE})=-3.37(1.77) \mathrm{mmHg}, 95 \% \mathrm{CI}=[-6.89 ; 0.16])$.

- FAS ISH: $-5.32 \pm 9.10 \mathrm{mmHg}$ versus $-6.40 \pm 7.40 \mathrm{mmHg}$, respectively $(\mathrm{E}(\mathrm{SE})=0.71(1.91) \mathrm{mmHg}$, $95 \% \mathrm{CI}=[-3.11 ; 4.53])$.

\section{- Office standing SBP/DBP at 3 min}

The decrease in the office standing SBP/DBP at 3 min was similar in both treatment groups over W0-W12:

- SBP: $-19.6 \pm 15.3 \mathrm{mmHg}$ versus $-21.9 \pm 16.4 \mathrm{mmHg}$, respectively $(\mathrm{E}(\mathrm{SE})=1.50(2.31) \mathrm{mmHg}$, $95 \% \mathrm{CI}=[-3.07 ; 6.07])$.

- DBP :

- FAS SDH: $-12.8 \pm 10.7 \mathrm{mmHg}$ versus $-10.7 \pm 9.4 \mathrm{mmHg}$, respectively $(\mathrm{E}(\mathrm{SE})=-2.42(1.95)$ $\mathrm{mmHg}, 95 \% \mathrm{CI}=[-6.31 ; 1.46])$.

- FAS ISH: $-5.1 \pm 10.5 \mathrm{mmHg}$ versus $-8.8 \pm 11.5 \mathrm{mmHg}$, respectively (E (SE) = $1.80(2.36) \mathrm{mmHg}$, $95 \% \mathrm{CI}=[-2.91 ; 6.52])$.

\section{- Office supine Mean BP (MBP)}

In the same way, a large decrease in the office supine MBP was observed in SDH patients:

- FAS SDH: -17.21 $\pm 10.26 \mathrm{mmHg}$ versus $-14.98 \pm 8.80 \mathrm{mmHg}$, respectively (E (SE) $=-2.61(1.86) \mathrm{mmHg}, 95 \% \mathrm{CI}=[-6.30 ; 1.08])$.

- FAS ISH: $-10.02 \pm 8.99 \mathrm{mmHg}$ in the Ind/Aml group versus $-11.70 \pm 7.82 \mathrm{mmHg}$ in the Ind + Aml group $(\mathrm{E}(\mathrm{SE})=1.48(2.04) \mathrm{mmHg}, 95 \% \mathrm{CI}=[-2.60 ; 5.57])$.

\section{- Office supine pulse pressure (PP)}

On the contrary, the office supine PP, which is calculated as the office supine SBP minus supine DBP, showed a greater decrease in ISH than SDH patients:

- FAS SDH: $-6.05 \pm 9.93 \mathrm{mmHg}$ versus $-9.75 \pm 10.87 \mathrm{mmHg}$, respectively $(\mathrm{E}(\mathrm{SE})=2.38(1.70) \mathrm{mmHg}$, $95 \% \mathrm{CI}=[-1.01 ; 5.77])$.

- FAS ISH: $-14.11 \pm 11.51 \mathrm{mmHg}$ versus $-15.89 \pm 14.64 \mathrm{mmHg}$, respectively (E (SE) $=2.09(3.01) \mathrm{mmHg}, 95 \% \mathrm{CI}=[-3.91 ; 8.10])$.

\section{- Blood pressure control and response to treatment}

The blood pressure control (defined by the percentage of patients with $\mathrm{SBP}<140 \mathrm{mmHg}$ and $\mathrm{DBP}$ $<90 \mathrm{mmHg}$ ) was reached by a large part of the patients at the last post-baseline value at W12, in each treatment group: $68.8 \%$ in the Ind/Aml group versus $63.6 \%$ in the Ind + Aml group (E (SE) $=5.19(7.61 \%)$, $95 \% \mathrm{CI}=[-9.72 ; 20.11])$.

In the same way, most of the patients responded to treatment at W12 (defined as the percentage of patients that reached a SBP $<140 \mathrm{mmHg}$ and $\mathrm{DBP}<90 \mathrm{mmHg}$, or SBP decrease $\geq 20 \mathrm{mmHg}$ from baseline, or DBP decrease $\geq 10 \mathrm{mmHg}$ from baseline): $75.3 \%$ in each treatment group, $\mathrm{E}(\mathrm{SE})=0.00(6.95) \%, 95 \% \mathrm{CI}$ $=[-13.62 ; 13.62]$. 


\section{SUMMARY - CONCLUSIONS (Cont'd)}

\section{SAFETY RESULTS}

\section{- Emergent adverse events}

The Table hereafter summarises the main results of adverse events in the Safety Set.

Overall summary for adverse events over the overall study in the Safety Set

\begin{tabular}{lccc}
\hline & & $\begin{array}{c}\text { Ind/Aml } \\
(\mathbf{N}=\mathbf{7 7})\end{array}$ & $\begin{array}{c}\text { Ind + Aml } \\
\text { (N = 77) }\end{array}$ \\
\hline Patients having reported at least one: & & & \\
$\quad$ Emergent adverse event & $\mathrm{n}(\%)$ & $18(23.4)$ & $14(18.2)$ \\
$\quad$ Treatment-related emergent adverse event & $\mathrm{n}(\%)$ & $8(10.4)$ & $6(7.8)$ \\
$\quad$ Emergent hypotension & $\mathrm{n}(\%)$ & $1(1.3)$ & $1(1.3)$ \\
$\quad$ Emergent orthostatic hypotension & $\mathrm{n}(\%)$ & $1(1.3)$ & $1(1.3)$ \\
$\quad$ Serious emergent adverse event & $\mathrm{n}(\%)$ & $3(2.6)$ & $2(2.6)$ \\
$\quad$ Treatment-related serious adverse event & $\mathrm{n}(\%)$ & - & - \\
Patients with treatment withdrawal due to & & & $1(1.3)$ \\
$\quad$ Emergent adverse event & $\mathrm{n}(\%)$ & $1(1.3)$ & - \\
$\quad$ Emergent serious adverse event & $\mathrm{n}(\%)$ & - & $1(1.3)$ \\
$\quad$ Treatment-related emergent adverse event & $\mathrm{n}(\%)$ & $1(1.3)$ & - \\
$\quad$ Treatment-related emergent serious adverse event & $\mathrm{n}(\%)$ & - & - \\
Patients who died & $\mathrm{n}(\%)$ & - & \\
\hline N: Number of patients by group & & & \\
n: number of affected patients & & & \\
$\%:(n * 100 / N)$ & & &
\end{tabular}

Emergent adverse events (EAEs) (considering all treatment doses received during the study) were reported with similar frequency in both treatment groups: $18 / 77$ patients, $23.4 \%$ in the Ind/Aml group versus 14/77 patients, $18.2 \%$ in the Ind + Aml group, in the Safety Set. The SOCs most frequently represented were:

- In the Ind/Aml group:

- Investigations: $9 / 77$ patients, $11.7 \%$ in the Ind/Aml group versus $3 / 77$ patients, $3.9 \%$ in the Ind + Aml group.

- Infections and infestations: $8 / 77$ patients, $10.4 \%$ versus $5 / 77$ patients, $6.5 \%$, respectively.

- In the Ind + Aml group:

- Infections and infestations (see above).

- Metabolism and nutrition disorders: $2 / 77$ patients, $2.6 \%$ versus $5 / 77$ patients, $6.5 \%$, respectively.

Most frequently reported EAEs (in more than 2 patients in any treatment group) on the overall study period were:

- In the Ind/Aml group:

- Glomerular filtration rate decreased (4/77 patients, $5.2 \%$ versus $1 / 77$ patients, $1.3 \%$, respectively), and blood uric acid increased and respiratory tract infection (3/77 patients, 3.9\% each preferred term versus none in the Ind/Aml group),

- In the Ind + Aml group: hyperuricaemia, 5/77 patients, $6.5 \%$ (versus none in the Ind/Aml group).

The other EAEs (oedema peripheral, hypokalaemia, hypotension) in patients treated with indapamide and amlodipine listed in the Summary of Product Characteristics were reported in few patients during the study:

- Oedema peripheral in 2 patients in the Ind/Aml group and 1 patient in the Ind+Aml group (of which one patient in the Ind/Aml group was treated at dose 2),

- Hypokalaemia in one patient in the Ind/Aml group only (at dose 2),

- Hypotension orthostatic in one patient in each treatment group (at dose 1). These orthostatic hypotensions were not serious, resolved, and the investigator specified that they were asymptomatic. One of them (in the Ind/Aml group) was considered as treatment-related by the investigator.

Most of the EAEs were rated mild (76.9\%) and none was severe. 


\section{SUMMARY - CONCLUSIONS (Cont'd) \\ SAFETY RESULTS (Cont'd)}

Treatment-related EAEs were reported in $8 / 77$ patients, $10.4 \%$ in the Ind/Aml group versus $6 / 77$ patients, $7.8 \%$ in the Ind + Aml group. They included mainly glomerular filtration rate decreased in the Ind/Aml group (3/77 patients, $3.9 \%$ versus $1 / 77$ patient, $1.3 \%$, in the Ind + Aml group), and hyperuricaemia in the Ind + Aml group (3/77 patients, $3.9 \%$ only reported in the Ind + Aml group). None of the other treatment-related EAEs were reported in more than 2 patients. None of the treatment-related EAEs was serious, or led to study drug withdrawal.

Few EAEs, all not-serious, led to patient's treatment withdrawal in 1/77 patients in the Ind/Aml group (alanine aminotransferase increased and aspartate aminotransferase increased, while the patient received the dose 1, i.e. Ind $1.5 \mathrm{mg} / \mathrm{Aml} 5 \mathrm{mg}$ ) and 1/77 patients in the Ind+Aml group (muscle spasms while the patient received the dose 2, i.e. Ind $1.5 \mathrm{mg}+\mathrm{Aml} 10 \mathrm{mg}$ ). All these EAEs were considered as treatment-related and were resolved.

Most of the EAEs (60.6\%) recovered or were recovering in the Ind/Aml group, whereas $47.4 \%$ of the EAEs recovered or were recovering in the Ind + Aml group.

Over the overall study period, 5/154 patients, 3.2\% reported 7 serious EAEs (including the Sponsor's upgrade), that were all emergent: $3 / 77$ patients, $3.9 \%$ in the Ind/Aml group reported 4 serious AEs (pneumonia, hypersensitivity, and in one patient: dyspnoea and platelet count decreased), and $2 / 77$ patients, $2.6 \%$ in the Ind + Aml group reported 3 serious AEs (pyelonephritis acute in one patient and one patient with both long QT syndrome and renal impairment). None of these serious EAEs was considered as treatment-related by the investigator or led to study withdrawal

None of the patients died during the study.

Regarding the analysis of the EAEs in patients that received dose 1 and those that received dose 2, similar safety profiles were obtained.

\section{Laboratory tests}

Neither clinically relevant change over time nor relevant between-group difference was observed for biochemistry and haematological parameters, in the Safety Set. Few PCSAs values for biochemistry were reported in both treatment groups. Most frequent PCSAs were detected for LDL cholesterol (high value) in 5 patients, $6.7 \%$ in the Ind/Aml group versus 3 patients, $4.0 \%$, in the Ind $+\mathrm{Aml}$ group, and creatinine Clearance (Cockroft formula) in 4 patients, $5.2 \%$ versus 5 patients, $6.5 \%$, respectively. The other PCSAs (high glucose, ALAT, and GGT) affected one patient each in the Ind/Aml group. Among the PCSAs detected, adverse events were reported for glucose (high value) in one patient in the Ind/Aml group (not serious, not treatment-related, not resolved), ALAT (high value) in one patient in the Ind/ Aml group (treatment-related, and resolved), and GGT (high value) in one patient in the Ind/Aml group (not treatment-related, and not resolved). For haematology, PCSA low value was reached for platelet in one patient in the Ind/Aml group and PCSA high value for eosinophils in one patient in the Ind + Aml group.

\section{Other safety evaluation}

For vital signs neither relevant change over time nor between-group difference was observed.

Emergent calculated orthostatic hypotension (according to a mathematic rule as a reduction of at least $20 \mathrm{mmHg}$ for SBP and/or $10 \mathrm{mmHg}$ for DBP at 1 and 3 minutes after standing-up compared to supine position, detected at post-baseline visit but not present at baseline) were detected in 2 patients, $2.6 \%$ in the $\mathrm{Ind} / \mathrm{Aml}$ group (one at $\mathrm{W} 6$ and one at $\mathrm{W} 12$ ) versus 1 patient, $1.3 \%$ in the Ind + Aml group (at W12).

One ECG abnormality was considered as clinically significant in one patient in each treatment group at W12 and reported as adverse event (hypokalaemia considered by the investigator as consistent with repolarisation changes, with no clinical manifestation in the Ind/Aml group and long QT syndrome, without any clinical symptoms in the Ind + Aml group). 


\section{CONCLUSION}

This international open Phase III study, conducted in 154 patients, aimed to demonstrate the effect of the fixed-dose Indapamide SR /Amlodipine in single-pill versus free dual therapy at the same dose, in lowering office supine systolic blood pressure, in patients with essential uncontrolled hypertension (previously treated or not). Over 12-week of treatment, a large decrease in the office supine SBP was obtained, similar in both treatment groups. A large decrease was already observed at 6 weeks of treatment. The titration, performed in patients not controlled at W6, allowed an important decrease on the office supine SBP. At week 12, most of the patients in both treatment groups had an office supine SBP controlled $(68.8 \%$ in the Ind/Aml group and $63.6 \%$ in the Ind $+A m l$ group) and a positive response to treatment for antihypertensive therapy $(\mathbf{7 5 . 3} \%$ in each treatment group). No relevant between-group difference was observed.

Secondary efficacy criteria yeldied similar trends.

Regarding safety, no unexpected adverse event was reported and the study showed no concern regarding the combination of indapamide and amlodipine. The safety was similar in the two treatment groups. The expected EAEs with indapamine and amlodipine (peripheral oedema, orthostatic hypotension, and hypokalaemia) were reported in few patients and remained low even with the lighest dose.

Both, the single-pill combination or free dual therapy were efficient and well tolerated and their safety profiles were in accordance with the known Summary of the Product Characteristics.

Date of the report: 24 September 2015

Version of the report: Final version 